\title{
Marker-Based Human Motion Capture in Multiview Sequences
}

\author{
Cristian Canton-Ferrer, Josep R. Casas, and Montse Pardàs \\ Signal Theory and Communications Department (TSC), Universitat Politècnica de Catalunya (UPC), Campus Nord, \\ Edif. D5, Jordi Girona 1-3, 08034 Barcelona, Spain
}

Correspondence should be addressed to Cristian Canton-Ferrer, cristian.canton@upc.edu

Received 24 March 2010; Accepted 6 November 2010

Academic Editor: Jar Ferr Yang

Copyright () 2010 Cristian Canton-Ferrer et al. This is an open access article distributed under the Creative Commons Attribution License, which permits unrestricted use, distribution, and reproduction in any medium, provided the original work is properly cited.

\begin{abstract}
This paper presents a low-cost real-time alternative to available commercial human motion capture systems. First, a set of distinguishable markers are placed on several human body landmarks, and the scene is captured by a number of calibrated and synchronized cameras. In order to establish a physical relation among markers, a human body model is defined. Markers are detected on all camera views and delivered as the input of an annealed particle filter scheme where every particle encodes an instance of the pose of the body model to be estimated. Likelihood between particles and input data is performed through the robust generalized symmetric epipolar distance and kinematic constrains are enforced in the propagation step towards avoiding impossible poses. Tests over the HumanEva annotated data set yield quantitative results showing the effectiveness of the proposed algorithm. Results over sequences involving fast and complex motions are also presented.
\end{abstract}

\section{Introduction}

Accurate retrieval of the configuration of an articulated structure from the information provided by multiple cameras is a field that found numerous applications in the recent years. The grown of computer graphics technology together with human motion capture (HMC) systems have been extensively used by the cinematographic and video games industry to generate virtual avatars [1]. Medicine also benefited from these advances in the field of orthopedics, locomotive pathologies assessment, or sports performance improvement [2]. In this field, despite markerless HMC systems have attained significant performance ratios in some scenarios [3], only HMC systems aided by markers placed on some body landmarks can produce high-accuracy results.

Depending on the type of employed markers, HMC systems are classified in two groups: nonoptical (inertial, magnetic, and mechanic) or optical systems (active and passive). Optical systems based on photogrammetric methods are more used than the nonoptical ones, usually requiring special suits embedding rigid skeletal-like structures [4], magnetic [5] or accelerometric devices [6] or multisensor fusion algorithms [7]. Instead, image-based or optical systems allow a relative freedom of movement and are less intrusive.
A common issue of all optical and nonoptical systems is the fact that they are usually expensive and require a dedicated hardware. The most usual involve IR retro-reflective markers that reflect back light, that is, generated near the cameras lens [8]. Other optical systems triangulate positions by using active markers that emits a pulse modulated signal. This allows distinguishing among markers and to automatically label them [9].

This paper focuses on HMC systems with passive markers in a multicamera scenario. These systems first require an accurate reconstruction of the markers' 3D position from its $2 \mathrm{D}$ projections which is not a trivial problem. Matches need to be established between the detected markers in the different views, defining the multiple view correspondences through homographies or algebraic methods [10]. This process is prone to errors due to occlusions, detection noise, and the proximity between markers. A temporal tracking of the markers also needs to be performed, to identify the markers in each sequence frame, thus yielding a 3D trajectory for each marker. Although professional systems exist for this purpose, errors occur when crucial markers become occluded or when markers' trajectories are confused. Finally, most applications require the transformation of the markers localization and trajectories to the motion parameters of a kinematic skeleton 
model. Commercial tools that perform this transformation are generally semiautomatic, thus becoming a labor-intensive task.

Once the 3D marker positions are obtained, it is required to fit a selected human body model (HBM) to these data to obtain kinematically meaningful parameters to perform either an analysis (i.e., for gesture recognition) or a synthesis (i.e., for avatar animation). However, in most of the systems, the markers' 3D position estimation and the fitting steps are decoupled. One of the first attempts to use an anatomical human model to increase the robustness of a HMC system is presented in [11] were the algorithm computes a skeletonand-marker model using a standardized set of motions and uses it to resolve the ambiguities during the $3 \mathrm{D}$ reconstruction process. Another approach using a HBM and data clustering is presented in [4]. Detection of 2D markers in separate images and its analysis using calibration information have been presented in [12] enforcing an HBM afterwards. A similar technique using a Kalman filter involving the HBM in the data association step was presented in [2].

In this paper, a low-cost real-time multicamera algorithm for marker-based human motion capture is presented. The proposed algorithm can work with any marker type detectable onto a set of $2 \mathrm{D}$ planes under perspective projection and it is robust to markers' occlusion and noisy detections. Since variables involved with the employed analysis HBM do not hold a linear relationship and the involved statistical distributions are non-Gaussian, we opted for a Monte Carlo approach to estimate the pose of the HBM at a given time instant. In our case, marker detection and HBM pose estimation are performed in the same analysis loop by means of an annealed particle filter [13]. Epipolar geometry is exploited in the particle likelihood evaluation by means of the symmetric epipolar distance [14] being robust to noisy marker detections and occlusions. Moreover, kinematic restrictions are applied in the particle propagation step towards avoiding impossible poses. Finally, effectiveness of the proposed algorithm is assessed by means of objective metrics defined in the framework of the HumanEva data set [3]. The presented algorithm is intended to work with any multicamera setup and regardless of the complexity of the selected human body model.

\section{Monte Carlo-Based Human Motion Capture}

2.1. Problem Formulation. The evolution of a physical articulated structure can be better captured with model-based tracking techniques [15]. In this process, the pose of an articulated HBM is sequentially estimated along time using video data from a number of cameras. Let $\mathbf{y}$ be the state vector to be estimated formed by the defining parameters of an articulated HBM, angles at every joint, and $y \subset \mathbb{R}^{D}$ the state space describing all possible valid poses an HBM may adopt, where $\mathbf{y} \in \mathcal{y}$.

From a Bayesian perspective, the articulated motion estimation and tracking problem is to recursively estimate a certain degree of belief in the state vector $\mathbf{y}_{t}$ at time $t$, given the data $\mathbf{z}_{1: t}$ up to time $t$. Thus, it is required to calculate the posterior $p d f p\left(\mathbf{y}_{t} \mid \mathbf{z}_{1: t}\right)$. However, this $p d f$ may be peaky and far from being convex, and hence cannot be computed analytically unless linear-Gaussian models are adopted. Even though Kalman filtering provides the optimal solution under certain assumptions, it tends to fail when the estimated probability density presents a multimodal distribution or the dimension of the state vector is high. Usually, this is the type of $p d f s$ involved in HMC processes.

2.2. Particle Filtering. Particle Filtering (PF) [16] algorithms are sequential Monte Carlo methods based on point mass (or "particle") representations of probability densities. These techniques are employed to tackle estimation and tracking problems where the $p d f$ s of the involved variables do not hold Gaussianity uncertainty models, linear dynamics and exhibit multimodal distributions. In this case, PF expresses the belief about the system at time $t$ by approximating the posterior probability distribution $p\left(\mathbf{y}_{t} \mid \mathbf{z}_{1: t}\right), \mathbf{y}_{t} \in \mathcal{Y}$. This distribution is represented by a weighted particle set $\left\{\left(\mathbf{y}_{t}^{j}, \pi_{t}^{j}\right)\right\}_{j=1}^{N_{p}}$, which can be interpreted as a sum of $N_{p}$ Dirac functions centered on the $\mathbf{y}_{t}^{j}$ with their associated real, nonnegative weights $\pi_{t}^{j}$ :

$$
p\left(\mathbf{y} \mid \mathbf{z}_{t}\right) \approx \sum_{j=1}^{N_{p}} \pi_{t}^{j} \delta\left(\mathbf{y}_{t}-\mathbf{y}_{t}^{j}\right) .
$$

In order to ensure convergence, weights must fulfill the normalization condition $\sum_{j} \pi_{t}^{j}=1$. For this type of estimation and tracking problems, it is a common approach to employ a Sampling Importance Resampling-(SIR)-based strategy to drive particles along time [17]. This assumption leads to a recursive update of the weights as

$$
\pi_{t}^{j} \propto \pi_{t-1}^{j} p\left(\mathbf{z}_{t} \mid \mathbf{y}_{t}^{j}\right)
$$

SIR PF circumvents the particle degeneracy problem by resampling with replacement at every time step [16]. That is, to dismiss the particles with lower weights and proportionally replicate those with higher weights. In this case, weights are set to $\pi_{t-1}^{j}=N_{p}^{-1}$, for all $j$, therefore

$$
\pi_{t}^{j} \propto p\left(\mathbf{z}_{t} \mid \mathbf{y}_{t}^{j}\right)
$$

Hence, the weights are proportional to the likelihood function that will be computed over the incoming data $\mathbf{z}_{t}$.

The best state at time $t, \hat{\mathcal{Y}}_{t}$, is derived based on the discrete approximation of (1). The most common solution is the Monte Carlo approximation of the expectation

$$
\widehat{y}_{t}=\mathrm{E}\left[p\left(\mathbf{y} \mid \mathbf{z}_{t}\right)\right]=\sum_{j=1}^{N_{p}} \pi_{t}^{j} \mathbf{y}_{t}^{j} .
$$

Usually, PF will be able to concentrate particles in the main mode of the likelihood function thus providing an estimation of the state space vector. However, multiple modes of similar size in the likelihood function might 
bias the estimation. In order to cope with such cases, the estimation is set to be the state vector associated to the maximum or the mean of all particle weights. Finally, a propagation model is adopted to add a drift to the state of the re-sampled particles in order to progressively sample the state space in the following iterations [16].

Another issue arising when applying PF techniques to computer vision problems is to derive a valid observation model $p\left(\mathbf{z}_{t} \mid \mathbf{y}_{t}^{j}\right)$ relating the input data $\mathbf{z}_{t}$ with the particle state $\mathbf{y}_{t}^{j}$. Nevertheless, even if such likelihood model can be defined, its evaluation may be very computationally inefficient. Instead of that, a fitness function $w\left(\mathbf{z}_{t}, \mathbf{y}_{t}^{j}\right)$ : $y \rightarrow[0,1]$ can be constructed according to the likelihood function, such that it provides a good approximation of $p\left(\mathbf{z}_{t} \mid \mathbf{y}_{t}^{j}\right)$ but is also relatively easy to calculate.

2.3. Annealing Strategy. PF is an appropriate technique to deal with problems where the posterior distribution is multimodal. This usually happens when state space dimensionality is high, like in HMC. To maintain a fair representation of $p\left(\mathbf{y}_{t} \mid \mathbf{z}_{1: t}\right)$, a certain number of particles is required in order to find its global maxima instead of a local one. It has been proved in [18] that the amount of particles required by a standard PF algorithm to achieve a successful tracking follows an exponential law with the number of dimensions. Articulated motion tracking typically employs state spaces with dimension $D>25$, thus standard PF turns out to be computationally unfeasible.

There exist several possible strategies to reduce the complexity of the problem based on refinements and variations of the seminal PF idea. Partitioned and hierarchical sampling $[18,19]$ are presented as highly efficient solutions to this problem. In the instance when there exists a tractable substructure between some variables of the state model, specific states can be marginalized out of the posterior, leading to the family of Rao-Blackwellized PF algorithms [20]. However, these techniques impose a linear hierarchy of sampling which may not be related to the true body structure assuming certain statistical independence among state variables. Finally, annealed PF [13] is one of the most general and robust approaches to estimation problems involving high-dimensional and multimodal state spaces. In this work, this technique will be extended to our markerbased scenario.

Likelihood functions $w\left(\mathbf{z}_{t}, \mathbf{y}\right)$ involved in HMC problems may contain several local maxima. Therefore, if using a single weighting function, a PF would require a large number of particles to properly sample the state space. By using annealing combined with $\mathrm{PF}$, a series of weighting functions $\left\{w_{m}\left(\mathbf{z}_{t}, \mathbf{y}\right)\right\}_{n=1}^{L}$ are constructed where $w_{m+1}\left(\mathbf{z}_{t}, \mathbf{y}\right)$ slightly differs from $w_{n}\left(\mathbf{z}_{t}, \mathbf{y}\right)$ and represents a smoothed version of it. In our case, $w_{L}\left(\mathbf{z}_{t}, \mathbf{y}\right)$ is designed to be a coarse smooth version of $w_{1}\left(\mathbf{z}_{t}, \mathbf{y}\right)$ and, typically, $w_{m}\left(\mathbf{z}_{t}, \mathbf{y}\right)$ functions are constructed by using

$$
w_{n}\left(\mathbf{z}_{t}, \mathbf{y}\right)=w\left(\mathbf{z}_{t}, \mathbf{y}\right)^{\beta_{n}},
$$

where $\beta_{L}<\cdots<\beta_{1}=1$ are the annealing scheduling parameters.

When a new measurement $\mathbf{z}_{t}$ is available an annealing iteration is performed. Every annealing run consists of $L$ steps or annealing layers where, in each of them, the appropriate weighting function is used and a set of pairs is constructed $\left\{\left(\mathbf{y}_{n, t}^{j}, \pi_{n, t}^{j}\right)\right\}_{j=1}^{N_{p}}$. Starting with an initialized particle set $\left\{\left(\mathbf{y}_{L, t}^{j}, \pi_{L, t}^{j}=N_{p}{ }^{-1}\right)\right\}_{j=1}^{N_{p}}$, the annealing process for every layer $n$ can be summarized as the following.

(1) Calculate the weights:

$$
\pi_{n, t}^{j} \propto w\left(\mathbf{z}_{t}, \mathbf{y}_{n, t}^{j}\right)^{\beta_{n}},
$$

enforcing the normalization condition $\sum_{j} \pi_{n, t}^{j}=1$. The estimation of parameter $\beta_{n}$ is based on the particle survival technique described in [13]. Once the weighted set is constructed, it will be used to draw the particles of the next layer.

(2) Resampling: draw $N_{p}$ particles with replacement from the set $\left\{\left(\mathbf{y}_{n, t}^{j}, \pi_{n, t}^{j}\right)\right\}_{j=1}^{N_{p}}$ with distribution $p(\mathbf{y}=$ $\left.\mathbf{y}_{n, t}^{j}\right)=\pi_{n, t}^{j}$.

(3) Construct the particle set corresponding to layer $n-1$ as

$$
\begin{gathered}
\mathbf{y}_{n-1, t}^{j}=\mathbf{y}_{n, t}^{j}+\mathcal{N}^{\star}\left(\mathbf{0}, \Sigma_{n}\right), \\
\pi_{n-1, t}^{j}=N_{p}^{-1},
\end{gathered}
$$

where $\mathcal{N}^{\star}\left(\boldsymbol{\mu}, \Sigma_{n}\right)$ stands for a truncated multivariate Gaussian distribution with mean $\mu$ and covariance matrix $\Sigma_{n}$ that will be further described in Section 3.5. This process is repeated until reaching $n=1$.

Finally the estimated state $\hat{y}_{t}$ is computed as

$$
\hat{y}_{t}=\sum_{j=1}^{N_{p}} \pi_{1, t}^{j} \mathbf{y}_{1, t}^{j} .
$$

The unweighted particle set for the next observation is defined as

$$
\mathbf{y}_{L, t+1}^{j}=\mathbf{y}_{1, t}^{j}+\mathcal{N}^{\star}\left(\mathbf{0}, \Sigma_{0}\right),
$$

where the covariance matrix $\Sigma_{0}$ is set proportional to the maximum variation of the defining model parameters and $\Sigma_{n}=\alpha^{L-m} \Sigma_{0}$. Setting $\alpha=0.6$ provided satisfactory results. A visual example of the annealed PF is depicted in Figure 1.

\section{Filter Implementation}

When implementing an annealed PF, several issues must be addressed: initialization, likelihood evaluation, particle propagation, and occlusion management. In the following section, we discuss the implementation of these two factors when employing a set of marker detections in multiple cameras as the input and an HBM as the tool to drive the physical relations among the variables of the state space (see Figure 2(a)). 


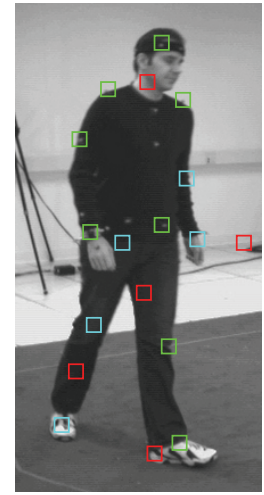

(a) Input

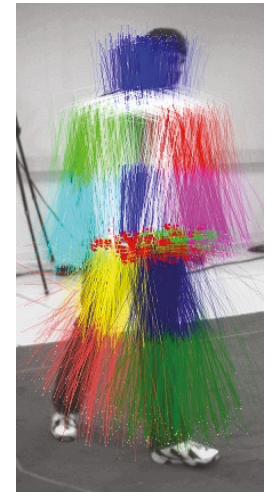

(1)

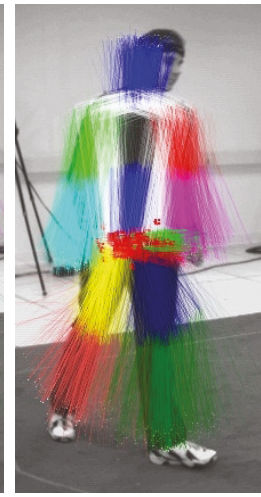

(b) Annealing PF
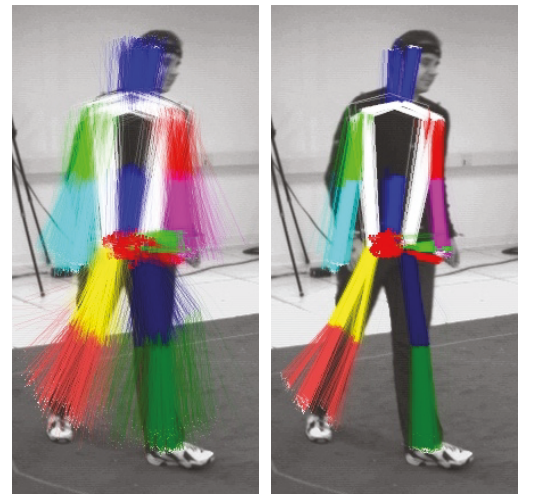

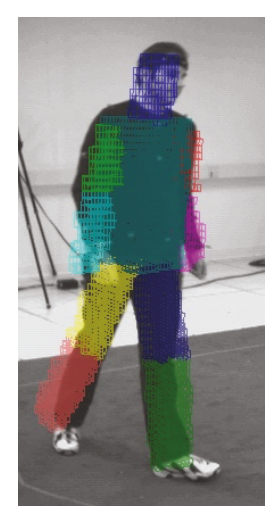

(c) Result

FIGURE 1: Annealed PF operation example. (a) The output of the employed marker detector where color boxes stand for correct (green), false (red), and missed (blue) detections. (b) The progressive fitting of particles driven by the annealing process and, (c) The final pose estimation $\hat{y}_{t}$.

3.1. Initialization. In the current scenario, it is supposed that the subject under study is tracked since the moment he/she enters the scene. A simple person tracking system is employed [21] to obtain a coarse estimation of person's position and velocity. Assuming that backward motions are unlikely, the velocity vector allows an initial estimation of the torso orientation. Finally, for the rest of limbs, a neutral and natural walking position is defined for the initialization of the HMC system.

In the case of a global miss of the tracked subject, the variance of the state space variables associated to every particle tend to be high in comparison of the variance obtained during a correct tracking operation. Therefore, the analysis of this variance allows detecting when the HMC system is out of track. In such case, the coarse tracking system is employed to start again the initialization loop described beforehand.

Although a beforehand selected HBM is employed to track any person, the size of the limbs must be adequate to the particular subject under study. For the majority of people, there is a strong quasilinear correlation between the height of a person and the length of the limbs [22] thus allowing a proper scaling of these magnitudes after automatically measuring the height directly from the input images as shown, for instance, in [14].

3.2. Measurement Generation. The input data $\mathbf{z}_{t}$ to the proposed tracking system will be the detection of the $2 \mathrm{D}$ projections of the set of distinguishable markers attached to the body of the performer onto the $N_{\mathrm{C}}$ available images in contrast with markerless HMC systems relying on image features such as edges or silhouettes [13]. Let $D_{n}=$ $\left\{\mathbf{d}_{1}, \mathbf{d}_{2}, \ldots, \mathbf{d}_{Q_{n}}\right\}$ be the set of $Q_{n}$ locations detected in the image captured in the $n$th view, $\mathbf{I}_{n}, 1 \leq n \leq N_{C}$. In order to generate $D_{n}$, a generic marker detection algorithm $\Gamma$ : $\mathbf{I}_{n} \rightarrow D_{n}$ is employed whose performance is assessed by the detection rate $(\overline{\mathrm{DR}})$, the false positive rate $(\overline{\mathrm{FP}})$, and the variance estimation error $\left(\sigma_{\Gamma}^{2}\right)$. This formulation of $\Gamma$ will allow performance comparisons of the tracking algorithm when using different marker detection algorithms and the assessment of occlusions.

Markers are usually placed at the joints, the end of the limbs, the top of the head and the chest of the subject. The proposed method is general enough to be applied to any type of markers detectable onto a set of $2 \mathrm{D}$ planes under perspective projection. An example of the detections obtained by our color-based marker detection is shown in Figure 2(b).

3.3. Likelihood Evaluation. In order to evaluate the likelihood between the body pose represented by a given particle state $\mathbf{y}_{t}^{j} \in \mathcal{y}$ with reference to the input data $\mathbf{z}_{t}=\left\{D_{n}\right\}_{n=1}^{N_{C}}$, a fitness function $w\left(\mathbf{z}_{t}, \mathbf{y}_{t}^{j}\right)$ must be defined. The $M 3 \mathrm{D}$ positions of the HBM landmarks corresponding to the pose described by the state vector $\mathbf{y}_{t}^{j}$ are computed through forward kinematics [12]. Let us denote these coordinates as the set $X=\left\{\mathbf{x}_{1}, \mathbf{x}_{2}, \ldots, \mathbf{x}_{M}\right\}, \mathbf{x}_{m} \in \mathbb{R}^{3}$. The fitness function relating the $3 \mathrm{D}$ locations set $X$ with the $2 \mathrm{D}$ observations $\left\{D_{n}\right\}_{n=1}^{N_{C}}$ should measure how well these $2 \mathrm{D}$ points fit as projections of the set $X$. A similar problem was tackled by the authors in [14] in a Bayesian framework and the underlying idea is applied in this context.

For every element $\mathbf{x}_{m} \in X$, its projection onto every camera is computed as

$$
\widetilde{\mathbf{p}}_{m, n}=\mathbf{P}_{n} \widetilde{\mathbf{x}}_{m}, \quad 1 \leq n \leq N_{C}
$$

where $\mathbf{P}_{n} \in \mathcal{M}_{4 \times 3}$ is the projection matrix associated to the $n$th camera [10] and tilde denotes homogeneous coordinates. Then, the set $T_{m}=\left\{\mathbf{t}_{1}, \mathbf{t}_{2}, \ldots, \mathbf{t}_{N_{\mathrm{C}}}\right\}$ containing the closest measurement in every camera view for every HBM landmark $\mathbf{x}_{m}$ is constructed as follows:

$$
\mathbf{t}_{n}=\min _{\mathbf{d}_{q} \in D_{n}}\left\|\mathbf{p}_{m, n}-\mathbf{d}_{q}\right\|, \quad \forall n .
$$

However, not all the 3D points $\mathbf{x}_{m}$ may have a projection onto every view due to occlusions or a miss-detection of the 


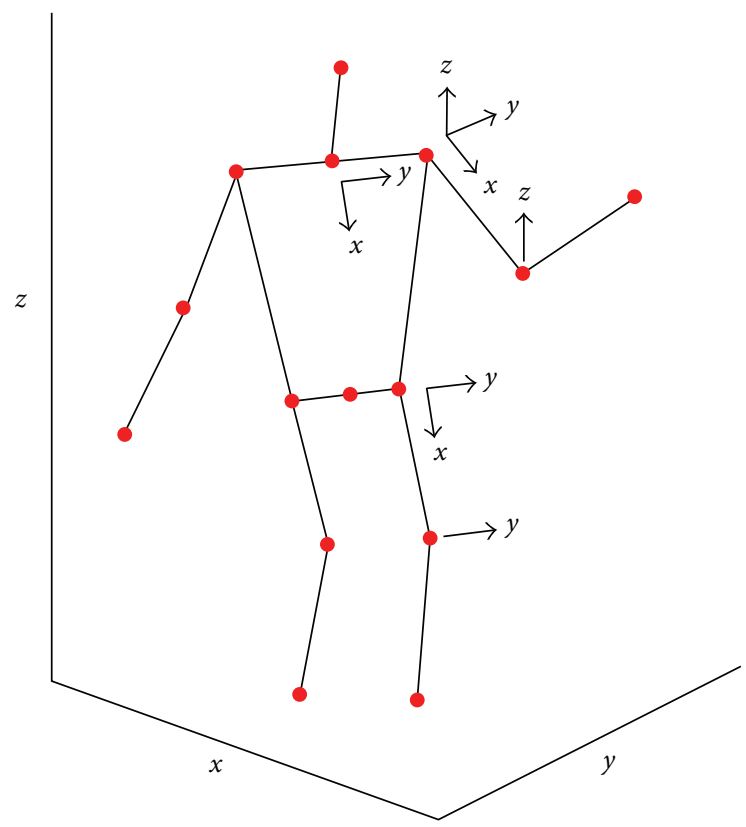

(a)

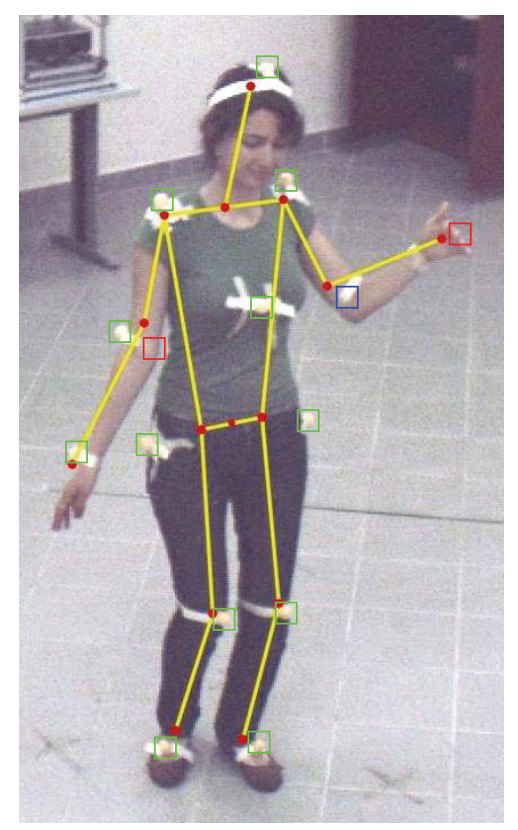

(b)

FIgURE 2: Human body model and measurement examples. In (a), the HBM employed in this paper is parameterized as follows: 2 DOF in the neck, 3 DOF in the shoulders, 1 DOF in the elbows, 3 DOF in the hips, 3 in the lower torso and 1 DOF in the knee. Red dots mark the HBM landmarks that can be computed by applying forward kinematics. In (b), the output of the employed color based marker location detection algorithm. Colors describe the correct detections (green), the miss detections (blue) and the false positive detections (red). All this detections will conform the measurement set $D_{n}$.

marker detection algorithm. In order to detect such cases, a thresholding is applied to the elements $\mathbf{t}_{n}$ dismissing those measurements above a threshold $\rho$. In this case, $\mathbf{t}_{n}=\varnothing$ using an empirically determined value of $\rho=10$ pixels. At this point, it is required measure how likely are the set of $2 \mathrm{D}$ measurements $T_{m}$ to be projections of the 3D HBM landmark $\mathbf{x}_{m}$. This can be done by means of the generalized symmetric epipolar distance $d_{\S \mathcal{E}}(\cdot)$ [14].

Let $l\left(\mathbf{x}^{i}, j\right)$ be the epipolar line generated by the point $\mathbf{x}$ in a given view $i$ onto another view $j$. Symmetric epipolar distance between two points $d_{\S \mathcal{E}}\left(\mathbf{x}^{i}, \mathbf{x}^{j}\right)$, in the two views $i, j$, is defined as

$$
d_{s \mathcal{E}}\left(\mathbf{x}^{i}, \mathbf{x}^{j}\right) \triangleq \sqrt{d^{2}\left(l\left(\mathbf{x}^{i}, j\right), \mathbf{x}^{j}\right)+d^{2}\left(l\left(\mathbf{x}^{j}, i\right), \mathbf{x}^{i}\right)},
$$

where $d\left(l\left(\mathbf{x}^{i}, j\right), \mathbf{x}^{j}\right)$ is defined as the Euclidean distance between the epipolar line $l\left(\mathbf{x}^{i}, j\right)$ and the point $\mathbf{x}^{j}$ as depicted in Figure 3. The extension of the symmetric epipolar distance for $k \geq 2$ points (in $k$ different views) $d_{s \mathcal{E}}\left(\mathbf{x}^{1}, \ldots, \mathbf{x}^{k}\right)$ can be written in terms of the distance defined in (12) as [14]

$$
d_{\S \mathcal{E}}\left(\mathbf{x}^{1}, \ldots, \mathbf{x}^{k}\right)=\sqrt{\sum_{i=1}^{k-1} \sum_{j=i+1}^{k} d_{\Im \mathcal{E}}^{2}\left(\mathbf{x}^{i}, \mathbf{x}^{j}\right)} .
$$

This distance produces low values when the $2 \mathrm{D}$ points are coherent, that is, when they are projections of the same $3 \mathrm{D}$ location. The score $s_{m}$ associated to $T_{m}$, and therefore to $\mathbf{x}_{m}$, is defined as

$$
s_{m}\left(\mathbf{z}_{t}, \mathbf{y}_{m}\right) \equiv s_{m}\left(\mathbf{z}_{t}, T_{m}\right) \propto d_{\S \mathcal{E}}\left(\mathbf{t}_{1}, \mathbf{t}_{2}, \ldots, \mathbf{t}_{N_{\mathrm{C}}}\right),
$$

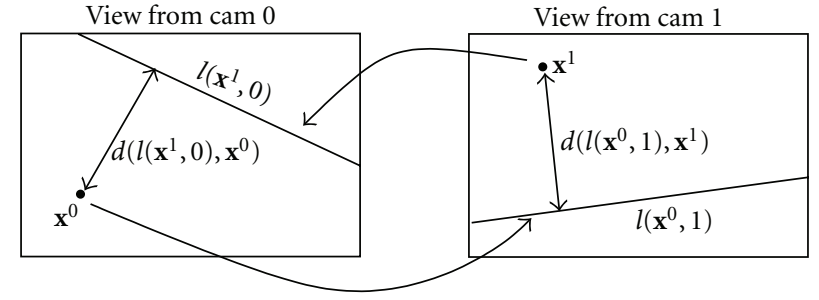

FIGURE 3: Symmetric epipolar distance between two points $d_{\S \mathcal{E}}\left(\mathbf{x}^{0}, \mathbf{x}^{1}\right)$.

and normalized such that $s_{m}\left(\mathbf{z}_{t}, T_{m}\right) \leq 1$. In the case where the nonempty elements of $T_{m}$ is below 2 , the distance $d_{s \mathcal{E}}\left(T_{m}\right)$ cannot be computed. Under these circumstances, we set $s_{m}\left(\mathbf{z}_{t}, T_{m}\right)=1$.

Assuming that the involved errors follow a Gaussian distribution [23], an accurate way to define the weighting function $w\left(\mathbf{z}_{t}, \mathbf{y}\right)$ is

$$
w\left(\mathbf{z}_{t}, \mathbf{y}\right)=\exp \left(-\frac{1}{M} \sum_{m=1}^{M} s_{m}\left(\mathbf{z}_{t}, \mathbf{x}_{m}\right)\right) .
$$

3.4. Occlusion Management. Occlusions are a major problem in HMC systems and can be separated into two categories: auto-occlusions and occlusions generated by opaque elements in the scene. In both cases, when analyzed from a multi-view perspective, occlusions are reflected in a missing subset of detected markers into some views. Assuming that 
there are $M$ markers attached to some HBM landmarks, the set $D_{n}$ would ideally contain the $M_{n} \leq M 2 \mathrm{D}$ projections of the markers that are not affected by the occlusions produced by the body itself onto the $n$th camera view. Moreover, there might be some miss-detections of these projection and a number of false measurements.

Within the current analysis framework, occlusions and miss-detections can be assumed as an underperformance of the generic marker detection $\Gamma$ thus regarded by the missdetection rate $\overline{\mathrm{DR}}$. As previously noted, the amount of false positives is represented by the false positive rate $\overline{\mathrm{FP}}$ and the error committed in the marker location estimation is assumed to have a Gaussian distribution with variance $\sigma_{\Gamma}^{2}$. This formation will allow simulating an arbitrary degree of corruption of the input data, as will be shown in Section 4.

Markers that are visible in, at least, three camera views can be correctly handled by the likelihood function. In the case of severe occlusions where there are only two camera views containing projections of a given marker, the distance $d_{\text {s\& }}$ may become inaccurate. In such cases, the position of the occluded marker is estimated using information from both the correctly estimated 3D neighboring landmarks and applying temporal coherence.

3.5. Propagation Model. Kinematic restrictions imposed by the angular limits at each joint of the HBM may produce a more robust tracking output. In this field, some methods employ large volumes of annotated data to accurately model the angular cross-dependencies among joints [24] or to learn dynamic models associated to a given action [25]. In our case, these angular constraints will be enforced in the propagation step of the APF scheme. Typically, the propagation step consists in adding a random component to the state vector of a particle as

$$
\mathbf{y}_{t}^{j}=\mathbf{y}_{t-1}^{j}+\mathcal{N}(\mathbf{0}, \mathbf{\Sigma})=\mathcal{N}\left(\mathbf{y}_{t-1}^{j}, \mathbf{\Sigma}\right) .
$$

That is, to generate samples from a multivariate Gaussian distribution centered at $\mathbf{y}_{t-1}^{j}$ with covariance matrix $\boldsymbol{\Sigma}$. However, this may lead to poses out of the legal angular ranges of the HBM. In order to avoid such effect, some works [26] add a term into the likelihood function that penalizes particles that do not fulfill the angular constraints. The following alternative is proposed to take into account angular constrains and draw samples from a truncated Gaussian distribution [27], denoted as $\mathcal{N}^{\star}$ and shown in Figure 4. In this way, particles are generated always within the allowed ranges thus avoiding the evaluation of particles that encode impossible poses and therefore increasing the performance of the sampling set.

\section{Experiments and Results}

4.1. Synthetic Data on HumanEva. In order to test the proposed algorithm, HumanEva data set [3] has been selected since it provides synchronized and calibrated data from both several cameras and a professional motion capture (MoCap) system to produce ground truth data. This data set contains a set of 5 actions performed by 3 different subjects captured by 4 fully calibrated cameras with a resolution of $640 \times 480$ pixels at $30 \mathrm{fps}$.

HumanEva suggests two metrics, mean, $\mu$, and standard deviation of the estimation error, $\sigma$, towards providing quantitative and comparable results. In this paper, metrics proposed in [28] for 3D human pose tracking evaluation are also employed. Let $X=\left\{\mathbf{x}_{1}, \mathbf{x}_{2}, \ldots, \mathbf{x}_{M}\right\}, \mathbf{x}_{m} \in \mathbb{R}^{3}$, denote the $M$ landmark positions of the HBM (typically, the body joints and the end of the limbs) corresponding to the pose described by the state variable $y \in \mathcal{y}$ computed using forward kinematics [12] at a given time $t$. Assuming that landmark positions $\hat{\mathbf{x}}_{m}$ associated to particle $\mathbf{y}^{j}$ are available, we can define a matched marker estimation $\hat{\mathbf{x}}_{m}$ with respect to the ground truth position $\mathbf{x}_{m}$ as the one fulfilling $\epsilon=\| \mathbf{x}_{m}-$ $\widehat{\mathbf{x}}_{m} \|<\delta$. This stands for those estimations that fall $\delta$-close to the ground truth position. Then, the Multiple Marker Tracking Accuracy (MMTA) is defined as the percentage of markers $\mathbf{x}_{m} \in X$ fulfilling the $\epsilon<\delta$ condition, and the Multiple Marker Tracking Precision (MMTP) as the average of the metric error between $\widehat{\mathbf{x}}_{m}$ and $\mathbf{x}_{m}$, of all pairs fulfilling $\epsilon<\delta$. Finally, these scores are averaged for all frames in the sequence. Threshold $\delta$, being an upper-bound of the maximum allowed error, is set to $\delta=100 \mathrm{~mm}$ in our experiments.

As it has been presented in Section 3.2, the input measurements $\mathbf{z}_{t}$ of the proposed algorithm are a set of 2D detections, $D_{n}$, measured over $N_{C}$ cameras for every time instant $t$. A synthetic data generation strategy has been devised where the $2 \mathrm{D}$ projection of the markers onto all camera views are computed from the $3 \mathrm{D}$ ground truth data, noted as $X_{t}$. This process is exemplified in Figure 5 and defined as follows.

(1) Inverse kinematics are applied to $X_{t}$ to estimate the pose of a HBM and body parts are fleshed out with super ellipsoids.

(2) Every 3D location in $X_{t}$ is projected onto every camera in order to generate the sets $D_{n}, 1 \leq n \leq N_{C}$. The previously estimated fleshed HBM checks the visibility of markers onto a given camera view by modeling the possible auto-occlusions among body parts. At this point, the $2 \mathrm{D}$ locations contained in $D_{n}$ are the positions obtained by an ideal marker detection algorithm.

(3) The effect of the marker detection algorithm $\Gamma$ is simulated by generating a number of miss detections, false measurements and, finally, adding a Gaussian noise to all measurements, according to the statistics reflected by $\overline{\mathrm{DR}}, \overline{\mathrm{FP}}$, and $\sigma_{\Gamma}^{2}$.

In order to test the performance of the proposed tracking algorithm, two factors must be taken into account: the performance of the marker detection algorithm $\Gamma$ (determined by the triplet $\left.\left\{\overline{\mathrm{DR}}, \overline{\mathrm{FP}}, \sigma_{\Gamma}^{2}\right\}\right)$ and the algorithm design parameters, that is, the number of layers $L$ and the number of particles per layer $N_{p}$. A simulation has been conducted testing a large number of combinations between parameters of $\Gamma$ and the proposed APF algorithm. The results of this 


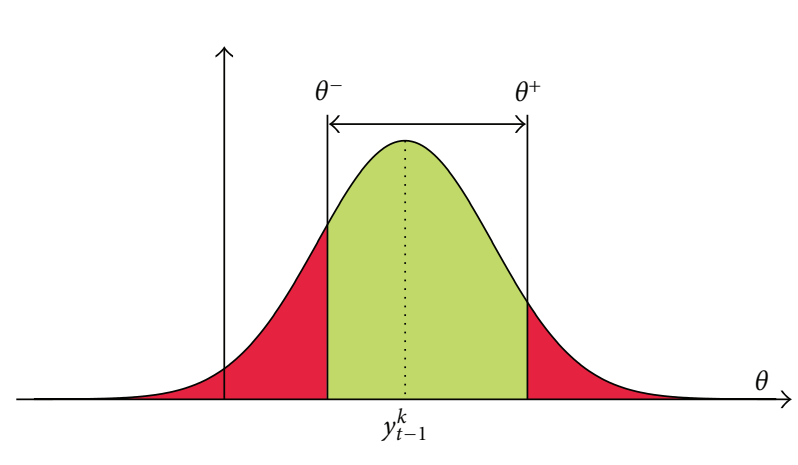

(a)

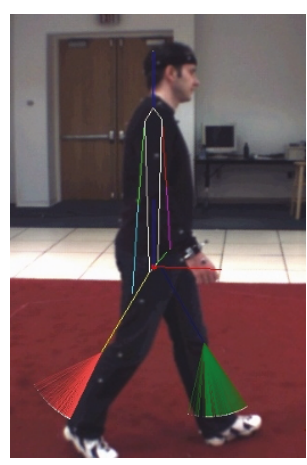

(b)

FIgURE 4: Angular constraints enforcement by propagating particles within the allowed angular ranges $\left[\theta^{-}, \theta^{+}\right]$. In $(\mathrm{a})$, samples are propagated following a truncated Gaussian distribution $\mathcal{N}^{\star}$ centered at $\mathbf{y}_{\mathbf{t}-1}^{j}$ with covariance matrix $\Sigma=\sigma$ bounded between $\theta^{-}$and $\theta^{+}$ (green zone). (b) An example of particle propagation in the knee angle displaying how propagated particles never fall out the legal ranges $(\theta<0)$.

simulation are depicted in Figure 6 where the MMTA score is displayed as the more informative metric [28].

When analyzing the impact of missing projections of markers, that is, occlusions, represented by $\overline{\mathrm{DR}}$ and shown in Figure 6(a), it can be seen that the algorithm is still robust producing accurate estimations even in the case of a large miss of data, $\overline{\mathrm{DR}}=0.4$. Assuming a fixed and realistic amount of occlusions, $\overline{\mathrm{DR}}=0.85$, we can explore the influence of the other distorting factors. Analyzing the results shown in Figure 6(b), it may be seen that the algorithm is robust against the number of false detections $\overline{\mathrm{FP}}$ since it is very unlikely that false $2 \mathrm{D}$ measurements in different views keep a 3D coherence. In this case, the spacial redundancy is efficiently exploited to discard these measurements. On the other hand, the performance of the algorithm decreases as the $2 \mathrm{D}$ marker position estimation error increases, $\sigma_{\Gamma}^{2}$. Another evident fact to be emphasized is the overannealing effect. The performance of the algorithm is not monotonically increasing with the number of employed annealing layers. This happens when the particles concentrate too much around the peaks of the weighting function hence impoverishing the overall representation of the likelihood distribution. For this motion tracking problem, we found that the optimal configuration is $L=3$ and $N_{p}=700$.

4.2. Real Data. The presented body tracking algorithm has been applied to capture motion figures from 4 different types of dances: salsa, belly dancing, and two Turkish folk dances. The analysis sequences were recorded with 6 fully calibrated cameras with a resolution of $1132 \times 980$ pixels at $30 \mathrm{fps}$.

Markers attached to the body of the dance performer were little yellow balls and a color-based detection algorithm $\Gamma$ has been used to generate the sets $D_{n}$ for every incoming multi-view frame. The original images are processed in the $\mathrm{YCrCb}$ color space which gives flexibility over intensity variations in the frames of a video as well as among the videos captured by the cameras from different views. In order to learn the chrominance information of the marker color, markers on the dancer are manually labeled in one frame
TABle 1: Result comparisons with state-of-the art methods evaluated over the HumanEva dataset. The presented score corresponds to the mean of the error estimation $\mu$, as reported by the compared authors in their respective contributions.

\begin{tabular}{|c|c|c|c|c|}
\hline Method & Walk & Jog & Box & Average \\
\hline $\begin{array}{l}\text { Hierarchical } \\
\text { Partitioned PF [26] }\end{array}$ & 101.9 & - & - & - \\
\hline $\begin{array}{l}\text { EM + Kinematically } \\
\text { constrained GMM } \\
{[29]}\end{array}$ & - & - & - & 150.9 \\
\hline $\begin{array}{l}\mathrm{PF}+\text { Dynamic } \\
\text { models [30] }\end{array}$ & 100.4 & - & - & - \\
\hline $\begin{array}{l}\text { ICP + Naïve } \\
\text { classification [31] }\end{array}$ & 53.1 & - & 45.4 & - \\
\hline $\begin{array}{l}\text { Example-based } \\
\text { pose estimation } \\
{[32]}\end{array}$ & 45.3 & 43.8 & 94.3 & - \\
\hline $\begin{array}{l}\text { Example-based } \\
\text { pose estimation }+ \\
\text { feature selection } \\
{[33]}\end{array}$ & - & - & - & 37.98 \\
\hline $\begin{array}{l}\text { Sparse probabilistic } \\
\text { regression [25] }\end{array}$ & 32.7 & 31.2 & 38.5 & - \\
\hline $\begin{array}{l}\text { Voxel } \\
\text { reconstruction }+ \\
\text { APF [34] }\end{array}$ & 96.5 & 130.34 & 145.22 & 121.18 \\
\hline Proposed method & 56.01 & 62.52 & 77.89 & 59.88 \\
\hline
\end{tabular}

for all camera views. It was assumed that the distributions of $\mathrm{Cr}$ and $\mathrm{Cb}$ channel intensity values belonging to marker regions are Gaussian. Thus, the mean can be computed over each marker region (a pixel neighborhood around the labeled point). Then, a threshold in the Mahalanobis sense is applied to all images in order to detect marker locations. An empirical analysis showed that the detector $\Gamma$ had the following performance triplet: $\overline{\mathrm{DR}}=0.98, \overline{\mathrm{FP}}=4$, and $\sigma_{\mathscr{D}}^{2}=20 \mathrm{~mm}$. 


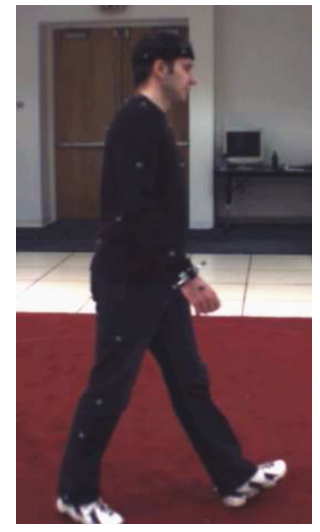

(a)

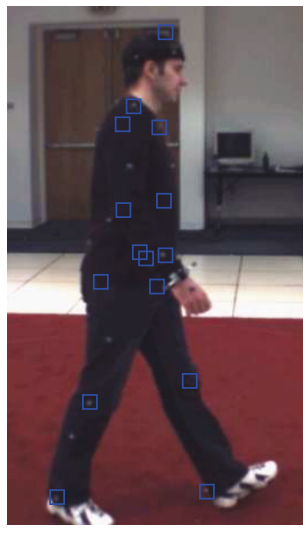

(b)

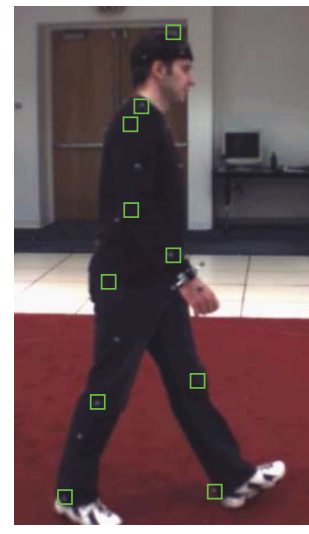

(c)

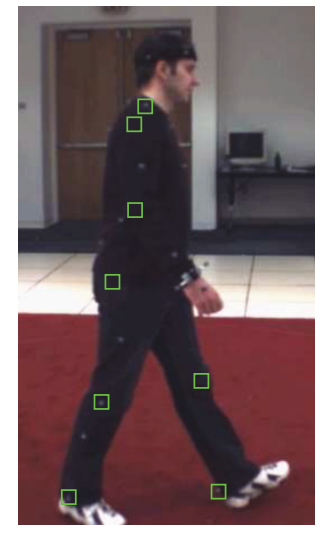

(d)

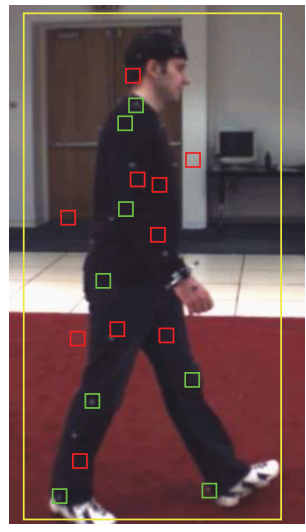

(e)

FIGURE 5: Synthetic data generation process. Since the reflective markers are not distinguishable in the original RGB image (a), the sets $\left\{D_{n}\right\}_{n=1}^{N_{C}}$ are generated from the $3 \mathrm{D}$ locations provided by the MoCap system. First, for a given view $n$, all $3 \mathrm{D}$ markers are projected onto the corresponding image (b), and those affected by body auto-occlusions are removed (c). Then, the marker detection algorithm $\Gamma$ is applied: some markers are missed due to the detection ratio (d), and a number of false measurements are generated (e). Finally, an amount of Gaussian noise with variance $\sigma_{\Gamma}^{2}$ is added simulating the position estimation error.

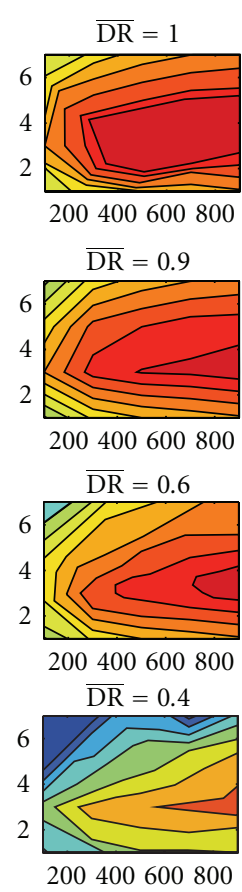

(a)

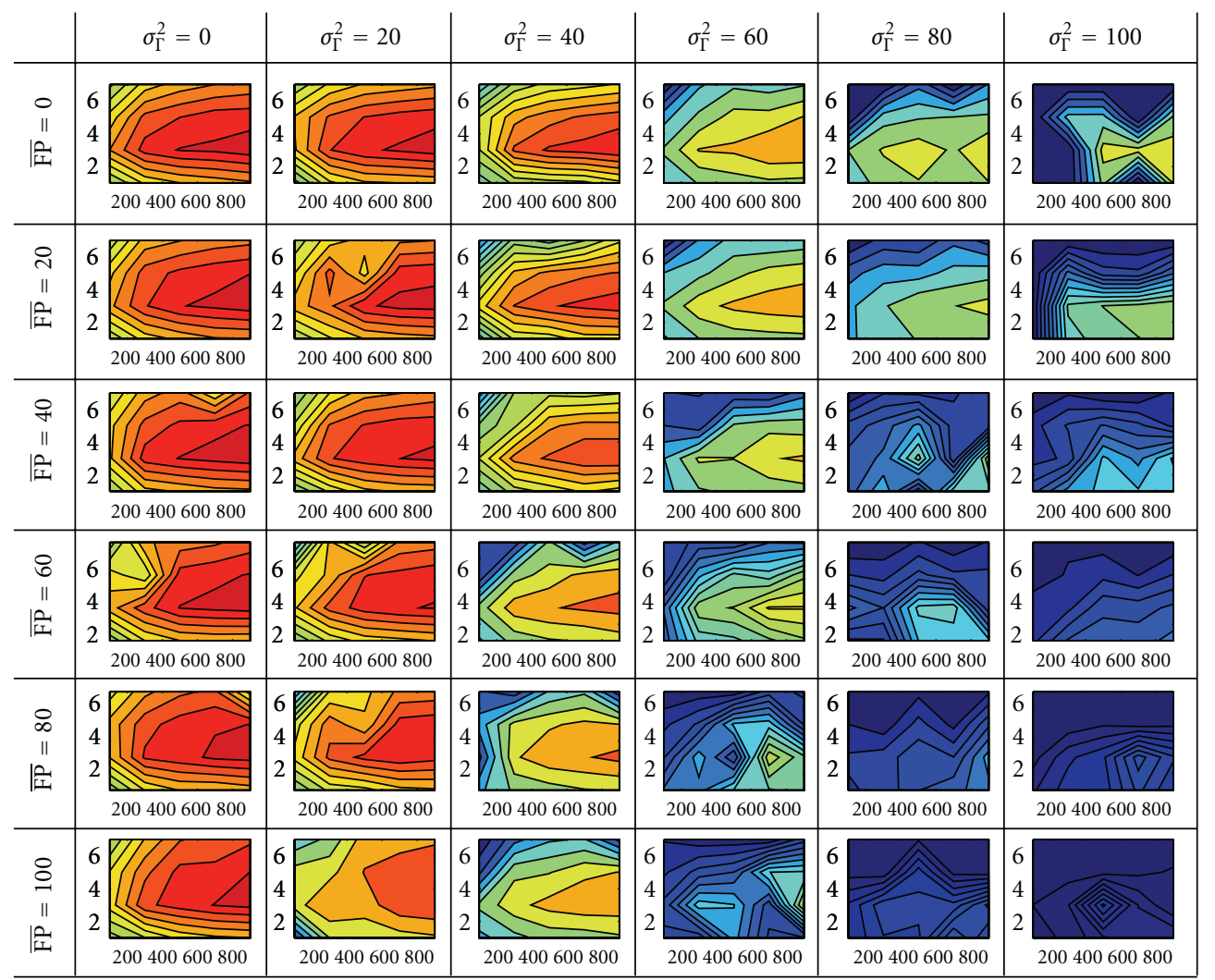

(b)

FIGURE 6: Quantitative results over the HumanEva data set where score MMTA is displayed in pseudocolor. In all plots, $y$-axis accounts for the number of layers $L$ and $x$-axis for the number of particles per layer $N_{p}$. In (a), assuming an ideal case where $\overline{\mathrm{FP}}=0$ and $\sigma_{\Gamma}^{2}=0$, impact of the number of occlusions, regarded by $\overline{\mathrm{DR}}$ in the overall performance. In (b), assuming a fixed occlusion level $\overline{\mathrm{DR}}=0.85$, results for the cases $\overline{\mathrm{FP}}=\{0,20,40,60,80,100\}$ and $\sigma_{\Gamma}^{2}=\{0,20,40,60,80,100\} \mathrm{mm}$. 

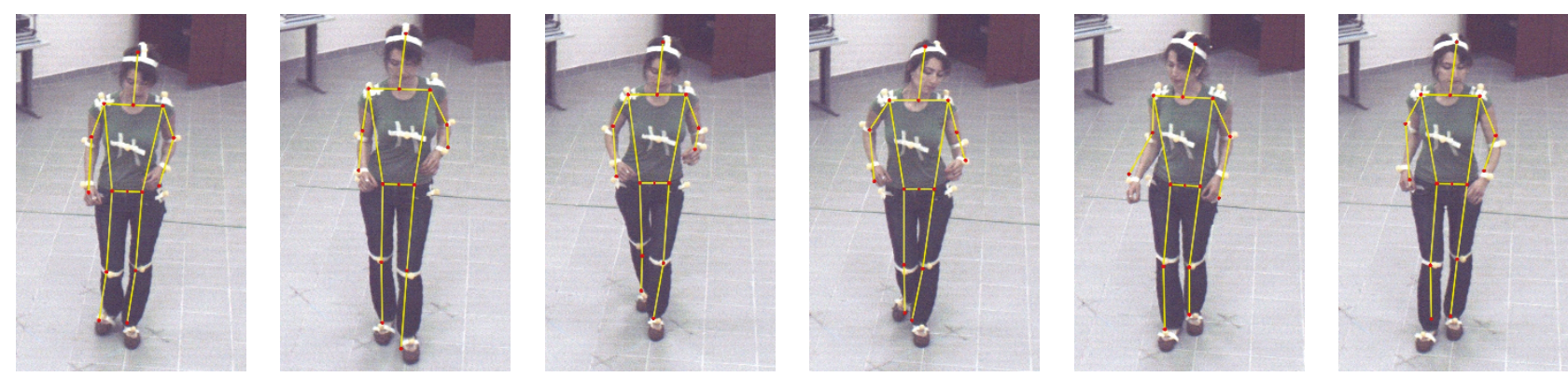

(a) Salsa figures
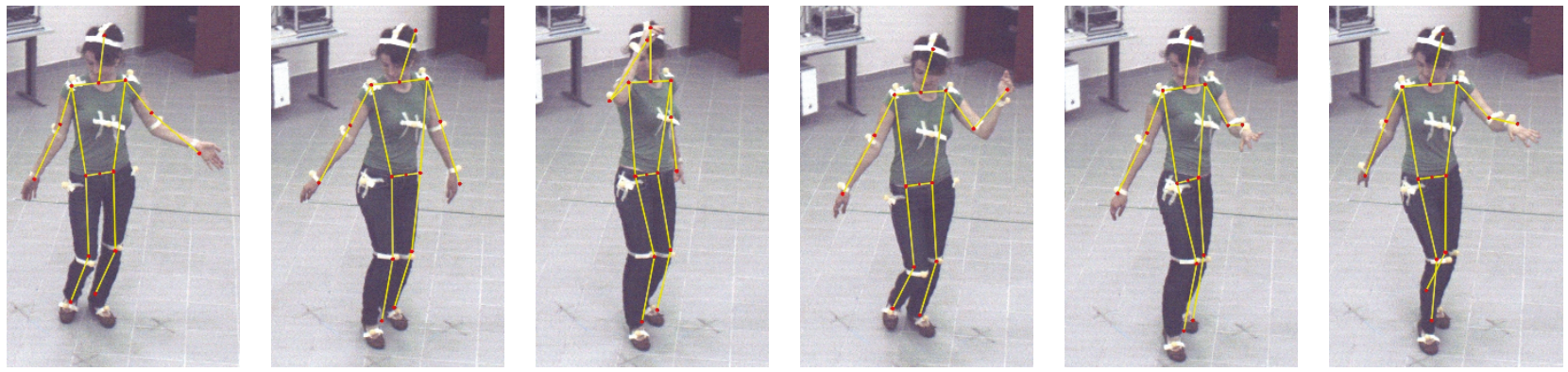

(b) Belly dancing figures

FIgURE 7: Dance motion tracking results. Two examples of dance tracking: salsa and belly dancing.

In this particular scenario, the algorithm had to cope with very fast motion associated to some figures. Even though these harsh conditions, the results were satisfactory and visually accurate as shown in Figure 7. Check http://www.cristiancanton.org/ for some example videos.

4.3. Results Comparison. A number of algorithms in the literature have been evaluated using HumanEva-I and their results have been reported in Table 1. There are two main trends in pose estimation: methods based on a tracking formulation of the problem and methods based on statistical classification. The method presented in this paper falls into the first category where some comparisons can be made. Among the reported methods, we find the expectation-maximization (EM) kinematically constrained GMM method presented by Cheng and Trivedi [29] as the continuation of the techniques already presented by Mikic [35]. Addressing a complex problem such as human motion capture using EM is perhaps manageable in a benevolent scenario with well learnt constrains but, as suggested by Caillete and Howard [36] in the comparison of EM- and PF- based methods, Monte Carlo-based techniques clearly outperform those based in minimization algorithms. Other contributions reported over HumanEva-I are based on the seminal idea of PF. Husz and Wallance [26] included a particle propagation step relying on learnt information on the structure of the executed motion thus facing the already mentioned problem of lack of adaptivity to unseen motions. A very detailed dynamic model of the human kinematics is employed by Brubaker et al. [30]. Motion involving a more complex pattern such as boxing or gesturing may not cope well with these two methods.
The other family of human motion capture algorithms is based on learning and classification instead of tracking. Basically, these techniques examine the ground truth data and extract a number of features from them. Afterwards, when a new test frame is processed, these same features are extracted, and the best match between them and the already learnt ones is outputted. Results obtained with these techniques, specially those of Urtasun et al. [25] and Poppe [32], outperform the tracking-based ones. However, these techniques are constrained to track a beforehand selected action and their applicability to unknown motion patterns is limited. It is notable the technique presented by Münderman et al. [31] where a 3D reconstruction is performed before computing the features to be learnt.

To the authors knowledge, there is no evaluation of a marker-based HMC system using the HumanEva dataset. The obtained results are close to those presented by classification-based markerless methods and, although the employed input data is different, it allows qualitatively evaluating its performance. An advantage of using a markerbased method is its robustness to faulty inputs, its low complexity, and the possibility of real-time implementations.

4.4. Real-Time Considerations. Once the image measurements have been obtained, the fitting of an HBM to these data using the proposed algorithm is achieved in real time in a $3 \mathrm{GHz}$ computer. Due to the low dimension of the input data $\left(\sum_{n=0}^{N_{C}} D_{n}\right)$, the computation of the involved operations in both the likelihood and propagation steps require a low computational cost. Measurements, can be obtained using elementary image filtering techniques as shown in 
Section 4.2 usually computed directly on the camera (as done by [8]) or by the digitizing hardware.

\section{Conclusion}

This paper presents a robust real-time low-cost approach to marker-based human motion capture using multiple cameras synchronized and calibrated. Progressive fitting of a human body model through the annealed particle filtering algorithm using a multi-view consistency likelihood function, the symmetric epipolar distance, and a kinematically constrained particle propagation model allow an accurate estimation of the body pose. Quantitative evaluation based on HumanEva dataset assessed the robustness of the algorithm when dealing faulty input data, even in very harsh conditions. Fast dance motion was also analyzed proving the adequateness of our technique to deal with a real scenario data.

\section{References}

[1] I. Baran and J. Popović, "Automatic rigging and animation of 3D characters," in Proceedings of the ACM International Conference on Computer Graphics and Interactive Techniques (SIGGRAPH '07), August 2007.

[2] P. Cerveri, A. Pedotti, and G. Ferrigno, "Robust recovery of human motion from video using Kalman filters and virtual humans," Human Movement Science, vol. 22, no. 3, pp. 377404, 2003.

[3] L. Sigal, A. O. Balan, and M. J. Black, "HumanEva: synchronized video and motion capture dataset and baseline algorithm for evaluation of articulated human motion," International Journal of Computer Vision, vol. 87, no. 1-2, pp. $4-27,2010$.

[4] A. G. Kirk, J. F. O’Brien, and D. A. Forsyth, "Skeletal parameter estimation from optical motion capture data," in Proceedings of IEEE Computer Society Conference on Computer Vision and Pattern Recognition (CVPR '05), pp. 782-788, June 2005.

[5] “Ascension," http://www.ascension-tech.com/.

[6] "Moven-inertial motion capture," http://www.moven.com/.

[7] D. Roetenberg, Inertial and magnetic sensing of human motion, Ph.D. dissertation, University of Twente, Twente, The Netherlands, 2006.

[8] "Vicon," http://www.vicon.com/.

[9] R. Raskar, H. Nii, B. Dedecker et al., "Prakash: lighting aware motion capture using photosensing markers and multiplexed illuminators," ACM Transactions on Graphics, vol. 26, no. 3, Article ID 1276422, 2007.

[10] R. Hartley and A. Zisserman, Multiple View Geometry in Computer Vision, C. U. Press, 2004.

[11] L. Herda, P. Fua, R. Plänkers, R. Boulic, and D. Thalmann, "Using skeleton-based tracking to increase the reliability of optical motion capture," Human Movement Science, vol. 20, no. 3, pp. 313-341, 2001.

[12] G. Guerra-Filho, "Optical motion capture: theory and implementation," Journal of Theoretical and Applied Informatics, vol. 12, no. 2, pp. 61-89, 2005.

[13] J. Deutscher and I. Reid, "Articulated body motion capture by stochastic search," International Journal of Computer Vision, vol. 61, no. 2, pp. 185-205, 2005.

[14] C. Canton-Ferrer, J. R. Casas, and M. Pardàs, "Towards a Bayesian approach to robust finding correspondences in multiple view geometry environments," in Proceedings of the 4th International Workshop on Computer Graphics and Geometric Modelling, vol. 3515 of Lecture Notes on Computer Science, pp. 281-289, 2005.

[15] T. B. Moeslund, A. Hilton, and V. Krüger, "A survey of advances in vision-based human motion capture and analysis," Computer Vision and Image Understanding, vol. 104, no. 2-3, pp. 90-126, 2006.

[16] M. S. Arulampalam, S. Maskell, N. Gordon, and T. Clapp, "A tutorial on particle filters for online nonlinear/non-Gaussian Bayesian tracking," IEEE Transactions on Signal Processing, vol. 50, no. 2, pp. 174-188, 2002.

[17] N. J. Gordon, D. J. Salmond, and A. F. M. Smith, "Novel approach to nonlinear/non-gaussian Bayesian state estimation," IEE Proceedings, Part F, vol. 140, no. 2, pp. 107-113, 1993.

[18] J. MacCormick and M. Isard, "Partitioned sampling, articulated objects, and interface-quality hand tracking," in Proceedings of the European Conference on Computer Vision, pp. 3-19, 2000.

[19] J. Mitchelson and A. Hilton, "Simultaneous pose estimation of multiple people using multiple-view cues with hierarchical sampling," in Proceedings of the British Machine Vision Conference, 2003.

[20] J. Madapura and B. Li, "3D articulated human body tracking using KLD-Annealed Rao-Blackwellised Particle filter," in Proceedings of IEEE International Conference onMultimedia and Expo (ICME '07), pp. 1950-1953, July 2007.

[21] C. Canton-Ferrer, J. R. Casas, M. Pardàs, and R. Sblendido, "Particle filtering and sparse sampling for multi-person 3D tracking," in Proceedings of IEEE International Conference on Image Processing (ICIP '08), pp. 2644-2647, October 2008.

[22] S. L. Dockstader, M. J. Berg, and A. M. Tekalp, "Stochastic kinematic modeling and feature extraction for gait analysis," IEEE Transactions on Image Processing, vol. 12, no. 8, pp. 962976, 2003.

[23] J. Lichtenauer, M. Reinders, and E. Hendriks, "Influence of the observation likelihood function on particle filtering performance in tracking applications," in Proceedings of the 6th IEEE International Conference on Automatic Face and Gesture Recognition (FGR '04), pp. 767-772, May 2004.

[24] L. Herda, R. Urtasun, and P. Fua, "Hierarchical implicit surface joint limits for human body tracking," Computer Vision and Image Understanding, vol. 99, no. 2, pp. 189-209, 2005.

[25] R. Urtasun, D. J. Fleet, and P. Fua, "3D people tracking with Gaussian process dynamical models," in Proceedings of IEEE Computer Society Conference on Computer Vision and Pattern Recognition (CVPR '06), pp. 238-245, June 2006.

[26] Z. Husz and A. Wallance, "Evaluation of a hierarchical partitioned particle filter with action primitives," in Proceedings of the 2nd Workshop on Evaluation of Articulated Human Motion and Pose Estimation, 2007.

[27] J. H. Kotecha and P. M. Djuric, "Gibbs sampling approach for generation of truncated multivariate Gaussian random variables," in Proceedings of IEEE International Conference on Acoustics, Speech, and Signal Processing (ICASSP '99), pp. 1757-1760, March 1999.

[28] C. Canton-Ferrer, J. Casas, M. Pardàs, and E. Monte, "Towards a fair evaluation of 3D human pose estimation algorithms," Tech. Rep., Technical University of Catalonia, 2009.

[29] S. Cheng and M. Trivedi, "Articulated body pose estimation from voxel reconstructions using kinematically constrained Gaussian mixture models: algorithm and evaluation," in 
Proceedings of the 2nd Workshop on Evaluation of Articulated Human Motion and Pose Estimation, 2007.

[30] M. Brubaker, D. Fleet, and A. Hertzmann, "Physics-based human pose tracking," in Proceedings of the Workshop on Evaluation of Articulated Human Motion and Pose Estimation, 2006.

[31] L. Münderman, S. Corazza, and T. Andriacchi, "Markerless human motion capture through visual hull and articulated icp," in Proceedings of the Workshop on Evaluation of Articulated Human Motion and Pose Estimation, 2006.

[32] R. Poppe, "Evaluating example-based pose estimation: experiments on the humaneva sets," in Proceedings of the 2nd Workshop on Evaluation of Articulated Human Motion and Pose Estimation, 2007.

[33] R. Okada and S. Soatto, "Relevant feature selection for human pose estimation and localization in cluttered images," in Proceedings of the European Conference on Computer Vision, 2008.

[34] C. Canton-Ferrer, J. R. Casas, and M. Pardàs, "Voxel based annealed particle filtering for markerless 3D articulated motion capture," in Proceedings of the 3rd IEEE Conference on 3DTV (3DTV-CON '09), May 2009.

[35] I. Mikič, Human body model acquisition and tracking using multi-camera voxel data, Ph.D. dissertation, University of California, San Diego, Calif, USA, 2003.

[36] F. Caillette and T. Howard, "Real-time markerless human body tracking with multi-view 3-D voxel reconstruction," in Proceedings of the British Machine Vision Conference, vol. 2, pp. 597-606, 2004. 